\title{
In silico comparasion of 11 different radioisotopes for palliative treatment of bone metastases
}

\author{
Guerra Liberal, F; Tavares João Manuel R. S. \\ Faculdade de Engenharia, Universidade do Porto \\ Porto, Portugal \\ meb12020@fe.up.pt; tavares@fe.up.pt
}

\author{
Tavares, A. \\ The Queens Medical Research Institute \\ University of Edinburgh \\ Edinburgh, Scotland, UK \\ adriana_tavares@msn.com
}

\begin{abstract}
Throughout the years, the palliative treatment of bone metastases using bone seeking radiotracers has been part of the therapeutic resources used in oncology. However, the choice of which bone seeking agent to use is not consensual. Computer simulation is a simple and practical solution to study and to compare a variety of radioisotopes. This study aimed to compare 11 different radioisotopes currently in use or under research. Hence, computational models were used to estimate the percentage of deoxyribonucleic acid damage, the probability of correct DNA repair, and the radiation-induced cellular effects post-irradiation. ${ }^{223} \mathrm{Ra}$ alpha particles, ${ }^{177} \mathrm{Lu}$ beta minus particles, and ${ }^{170} \mathrm{Tm}$ beta minus particles induced the highest cell death of all investigated particles and radioisotopes. The cell survival fraction measured post-irradiation with beta minus particles emitted by ${ }^{89} \mathrm{Sr}$ and ${ }^{153} \mathrm{Sm}$, was higher than ${ }^{177} \mathrm{Lu}$ beta minus particles and ${ }^{223} \mathrm{Ra}$ alpha particles. Findings reported here may prompt future in vitro and in vivo experiments comparing different radionuclides for palliative treatment of bone metastases.
\end{abstract}

Index Terms-computational methdos; deoxyribonucleic acid; Radium-223; Lutetium-177

\section{INTRODUCTION}

Cancer is a major public health concern, and it is associated with significant morbidity and mortality. In 2012, approximately 14 million new cases were diagnosed, and 8 million cancer-related deaths occurred. Prostate cancer is the second most common cancer in men worldwide [1]. A large percentage of patients with prostate cancer (65-75\%) develop bone metastasis[2]-[4], which often leads to severe pain, hypercalcemia, lack of mobility and depression that adversely affect these patients' life quality [5]. There are several therapeutic approaches targeting bone metastases and its associated effects, including the use of analgesics, external beam radiotherapy and radionuclide systemic therapy. The latter, systemic palliative targeted-therapy with suitable radiotracers, has emerged as a particularly appealing and efficient treatment modality for patients with multiple skeletal metastases [3], [6]-[8].

Over the years, efforts were made to identify radionuclides with improved physical properties for use in palliative care of metastatic bone pain. A major challenge associated with the palliative treatment of bone pain using selective radiotracers is to deliver an adequate dose of ionizing radiation to the bone lesion, while minimizing the dose to healthy bone sites and adjacent tissues. The radionuclide emitted particles should have an energy and tissue penetration range compatible with the volume of the lesion to be irradiated [2], [9], given that both the energy and the penetrating range of a radionuclide emitted particle significantly contribute to the therapy efficacy [10]. Therefore, studying the effects of particulate radiation at the cellular level is of interest to determine the suitability of a given radionuclide for target-tumor radiotherapy. There are essentially three main types of radioactive particles that are of interest for target-tumor radiotherapy: beta minus $\left(\beta^{-}\right)$particles, alpha $(\alpha)$ particles and Auger electrons. In addition to the physical properties of different radionuclides for target-tumor therapy, other characteristics should be taken into consideration while searching for the ideal radiotracer for palliative treatment of bone metastases. For example, the radionuclide production costs and feasibility should be determined, and consideration should be given on whether its properties are amenable for radiochemistry procedures [8].

${ }^{32} \mathrm{P}$ was the first radioisotope to be evaluated for palliative treatment of bone metastases and its first clinical use dates back to 1941 [11]. Presently, one of the most commonly used radionuclides for palliative treatment of bone metastases in routine clinical practice is ${ }^{153} \mathrm{Sm}$, which was approved by the Food and Drug Administration (FDA) for clinical use in 1997 [12]. ${ }^{89} \mathrm{Sr}$ is also currently used in the clinical setting for alleviation of bone pain and it was approved by the FDA for clinical use in 1993 [13]. Recently, a significant amount of work has been carried out using ${ }^{223} \mathrm{Ra}$, a promising therapeutic agent for palliative treatment of bone metastases, in order to take advantage of the low tissue penetration range associated with its emitted $\alpha$ particles. Its clinical use was approved by the FDA in 2013 [14]. Another promising radionuclide recently proposed for palliative treatment of bone metastasis was ${ }^{177} \mathrm{Lu}$, due to its appealing physical characteristics; in particular, its half-life of 6.73 days, gamma ray emission of $113 \mathrm{keV}(6.4 \%)$ and $208 \mathrm{keV}(10.4 \%)$ and tissue penetration of $1.8 \mathrm{~mm}$ [13].

Despite the increasing availability of radionuclides for palliative treatment of bone metastases [13], [15], the choice of which therapeutic agent to use is not consensual across sites

$4^{\text {th }}$ Portuguese Meeting in Bioengineering, February 2015

Portuguese chapter of IEEE EMBS

Faculty of Engineering of the University of Porto 
and no standardization or guideline exists to assist with this choice. This may be due to the limited data available concerning the advantages and disadvantages of each radioisotope, as well as assessing all radioisotopes in the same test conditions. Computational simulation is a simple and practical method to study and to compare a variety of radioisotopes for different medical applications, including the palliative treatment of bone metastases, under the same research conditions [16]. This study aimed to use computational methods to compare different radioisotopes currently in use or under research for the palliative treatment of bone metastases. In particular, the work developed is focused on the bone metastases from the prostate carcinoma, given this is one of the most common types of cancer in men worldwide, and the majority of these patients develop bone metastases.

\section{METHODS}

Three different computational simulators were used to investigate the therapeutic potential of the following radioisotopes: ${ }^{32} \mathrm{P},{ }^{89} \mathrm{Sr},{ }^{90} \mathrm{Y},{ }^{117 \mathrm{~m}} \mathrm{Sn},{ }^{153} \mathrm{Sm},{ }^{166} \mathrm{Ho},{ }^{170} \mathrm{Tm}$, ${ }^{177} \mathrm{Lu},{ }^{186} \mathrm{Re},{ }^{188} \mathrm{Re}$ and ${ }^{223} \mathrm{Ra}$. The sections below explain the main principles of these simulators and point out the key input parameters adopted.

\section{A. Monte Carlo damage simulator}

The Monte Carlo damage simulation (MCDS) algorithm can be used to predict the types of deoxyribonucleic acid (DNA) damage and their yield after irradiation. This method allows for rapid collection of a large data set with multiple irradiation scenarios. The model generates a number of damage configurations expected within the DNA of one cell, where the algorithm processes information in two main steps: first, it randomly distributes, in a DNA segment, the expected amount of damage produced in a cell; then, it subdivides the distribution of damage in that particular DNA segment. A detailed description of the MCDS model as well as additional discussions about the validity and limitations this method are presented in [17] and [18].

The classification scheme used by the MCDS to categorize DNA damages is based on the classification parameters proposed by Nikjoo et al., for further details, see [19] and [20].

\section{B. Monte carlo excision repair simulator}

The Monte Carlo excision repair (MCER) algorithm can be used to calculate the formation and repair of DNA damages and to predict repair outcomes such as correct repair, repair with a mutation and conversion into a DSB [21]. The MCER algorithm starts by using the MCDS algorithm to generate a random number of damage configurations expected within the DNA of one cell. Subsequently, the MCDS-generated damage configurations are superimposed over a predetermined nucleotide sequence or a random nucleotide sequence. Then, the MCER model is used to simulate the repair, misrepair and aborted excision repair of damage within the entire genome or within a specific region of the DNA. The lesions forming a cluster are removed sequentially through repeated rounds of excision repair.
The majority of DNA oxidative damage, including modified apurinic/apyrimidinic converted into strand breaks, requires repair by base excision repair (BER). Two different types of BER processes have been observed in eukaryotic and prokaryotic cells: (1) excision and replacement of a single nucleotide, known as short-patch BER (SP-BER), which occurs in most cases; and (2) replacement of 2-13 nucleotides, known as long-patch BER (LP-BER). Another enzymatically distinct repair pathway is nucleotide excision repair (NER). The NER, observed in eukaryotic cells, substitutes oligonucleotide fragments of 24-32 nucleotides in length [21]. Results from this simulator are outputted as simplified repair scenarios, due to the current uncertainties associated with the processing of radiation-induced damage by the BER and NER pathways. The studies by Semenenko and Stewart have demonstrated that the results obtained by this simulator correlate well with in vitro results obtained using cell cultures [22]. A detailed description of the MCER algorithm, as well as additional discussions on the validity and limitations of the model can be found in [17] and [21].

\section{Virtual Cell radiobiology simulator}

The radiation-induced damage can be lethal or can be slowly repaired via enzymatic repair and misrepair, which then determines the fate of the irradiated cell. The cell cycle kinetics can influence and/or be influenced by the kinetics of damage processing; and damages to the DNA are often a trigger for apoptosis, although cell membrane damages can also induce apoptosis [21], [23]. Radiobiological models have been developed to describe the dose-response association, the process of damage production and the key repair mechanisms. These models often correlate the dose rate with the cell response to irradiation.

Further details on this computational simulator can be found at: http://faculty.washington.edu/trawets/vc.

\section{Simulated parameters}

For each investigated radioisotope, only the most abundantly emitted radioactive particles were evaluated using the MCDS and MCER simulators. The general input details used for simulations with the MCDS model were as follows: initial cell number of 1000 and DMSO concentration of 0 . The MCER input conditions are defined as: inhibition distance of 3 base pairs; probability of choosing a lesion from the first stand break of 0.5; polymerase error rate for SP-BER and LP-BER of $1.0^{-4}$ and $1.0^{-6}$, respectively; probability of incorrect insertion of opposite base lost of 0.75 ; and probability of incorrect insertion of opposite damaged base of 0.75 .

In the input file for the $\mathrm{VC}$ simulator, the cell cycle time $\left(T_{c}\right)$ and the cell potential doubling time $\left(T_{p o t}\right)$ values were adjusted to modeling two different cell phenotypes: normal prostate cells $\left(T_{c}=48 \mathrm{~h}\right.$ and $T_{\mathrm{pot}}=500$ days $)$ and metastatic prostate cells $\left(T_{C}=48 \mathrm{~h}\right.$ and $T_{\text {pot }}=54$ days) [24]. The number of DSBs and the percentage of complex DSBs were obtained from the MCDS simulator. These results were then applied as input parameters for the TLK model used in the VC simulator. Irradiation periods of $2,24,48,120$ and $240 \mathrm{~h}$ (TCUT - time allowed for repair after exposure), with total 
absorbed doses of $0.1,0.5,1,2,5$ and 8 Gy were studied using the VC simulator. Other parameters used in the VC simulator, specified in the TLK model input file, included: (1) DRM (damage repair model) $=$ TLK, (2) CKM (cell kinetics model) = QECK (quasi-exponential cell kinetics model); (3) DNA (cell DNA content) $=5.667 \mathrm{D}+09$ base pair; $(4)$ DSB (endogenous) $=4.3349 \times 10^{-03} \mathrm{~Gy}^{-1}$ cell $^{-1}$; (5) RHT (repair halftime $)=\mathrm{XXX}, \mathrm{XXX}=0.25,9 \mathrm{~h}$ (simple DSBs are repaired faster than complex DSBs); (6) A0 (probability of correct repair $)=\mathrm{AAA}, \mathrm{AAA}=0.95,0.25$ (simple $\mathrm{DSBs}$ are repaired more accurately than complex DSBs); (7) ETA (pairwise damage interaction rate) $=2.5 \times 10^{-04} \mathrm{~h}^{-1} ;$ (8) PHI (probability of a misrejoined DSB being lethal) $=0.005$; (9) GAM (fraction of binary-misrepaired damages that are lethal) $=$ 0.25; (10) N0 (initial number of cells) $=1000$; (11) KAP (peak cell density) $=1.0 \mathrm{D}+38$ cells $/ \mathrm{cm}^{3} ;$ (12) VOL (tissue volume) $=1 \mathrm{~cm}^{3}$; (13) FRDL (fraction of residual that is lethal damage) $=0.5$; (14) ACUT (absolute residual damage cutoff $)=1.0 \mathrm{E}-09$ expected number of DNA damages per cell; (15) BGDR (average background absorbed dose rate on planet Earth) $=2.73748 \times 10^{-07} \mathrm{Gyh}$; (16) DCUT (dose cutoff) $=0.01 \mathrm{~Gy}$; (17) STOL (step-size tolerance) $=0.01 \mathrm{Gyh} ;(18)$ $\mathrm{SAD}($ scaled absorbed dose) $=\mathrm{RX} 1, \mathrm{RX} 1=0.1,0.5,1,2,5$ and $8 \mathrm{~Gy}$; (19) GF [growth fraction, if 0 (zero) all cells are quiescent, if 1 (one) all cells are cycling and if 0.5 the cell population is heterogeneous] $=0,0.5,1[25]$.

\section{RESUlTS}

\section{A. MCDS and MCER}

Fig 1 presents the radiation-induced DNA damages obtained post-irradiation with different radioactive particles, emitted by selected radioisotopes. The probability of correct repair, repair with a mutation and conversion into a DSB, and the number of repair cycles are presented in Fig 2. Results obtained using the MCDS and MCER algorithms showed that ${ }^{223} \mathrm{Ra} \alpha$ particles were able to induce the highest amount of DNA damages of all investigated particles, having the lowest probability of correct repair of damages and highest probability of the repair processes to induce DSB.

\section{B. VC simulator}

The cell survival fraction results obtained after irradiation with different radionuclides and particles are shown in Fig 3. The estimated survival fraction rank order of all investigated particles was: ${ }^{166}$ Ho $\beta^{-}$particles, ${ }^{186} \operatorname{Re} \beta^{-}$particles, ${ }^{188} \operatorname{Re} \beta^{-}$ particles $>{ }^{89} \mathrm{Sr} \beta^{-}$particles $>{ }^{32} \mathrm{P} \beta^{-}$particles $>{ }^{90} \mathrm{Y} \beta^{-}$ particles $>{ }^{117 \mathrm{~m}} \mathrm{Sn}$ electrons $>{ }^{153} \mathrm{Sm} \beta^{-}$particles $>{ }^{177} \mathrm{Lu} \beta^{-}$ particles $>{ }^{170} \mathrm{Tm} \beta^{-}$particles $>>{ }^{223} \mathrm{Ra} \alpha$ particles. Additionally, statistically significant differences were observed among the different irradiating agents $(p<0.0001$ and $p=0.0053$ ANOVA, with and without ${ }^{223} \mathrm{Ra}$ data, respectively). The ${ }^{223} \mathrm{Ra} \alpha$ particles, the ${ }^{170} \mathrm{Tm} \beta^{-}$particles and the ${ }^{177} \mathrm{Lu} \beta^{-}$particles had the lowest survival fraction of all investigated irradiating agents, as determined using the VC simulator. Detailed examination of the estimated number of surviving cells at different proliferation status for these 3 irradiating agents is presented in Figs. 4 and 5. The estimated number of normal prostate cells that survived irradiation when all cells were quiescent and when all cells were actively dividing is presented in Figs. $4 a-4 b$, respectively. The number of metastatic prostate cells that survive the irradiation when all cells are quiescent and all cells are actively dividing is shown in Figs. 5a-5b, respectively. When all cells were quiescent, the estimated number of normal prostate surviving cells is similar to the estimated number of metastatic prostate cells that survive irradiation and seems to be independent from the cell kinetic (Figs. 4a and 5a). Conversely, for actively dividing cellular populations, it is possible to observe that there are fewer surviving cells in the normal prostate cell population than in the metastatic prostate cell population (Figs. $4 \mathrm{~b}$ and $5 \mathrm{~b}$ ). The estimated number of cells that survived a given irradiation scenario decrease in the following order: ${ }^{177} \mathrm{Lu} \beta^{-}$particles $>{ }^{170} \mathrm{Tm} \beta^{-}$particles $>$ ${ }^{223} \mathrm{Ra} \alpha$ particles. Statistically significant differences were observed among these 3 different irradiating agents when normal prostate cells were quiescent and all cells were actively dividing ( $p<0.0001$, ANOVA). Similar results were found for the metastatic prostate cells $(p<0.0001$, ANOVA).
A
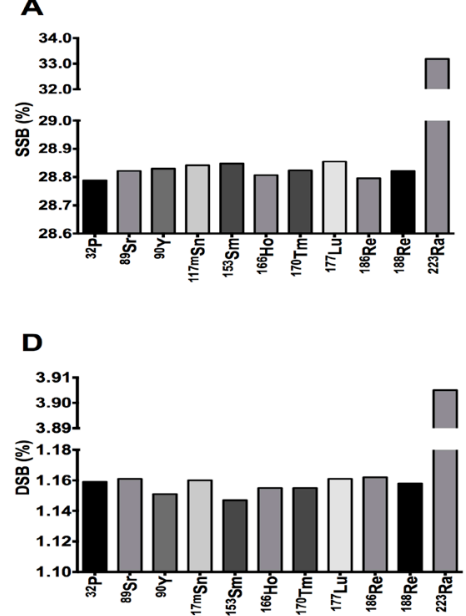

B

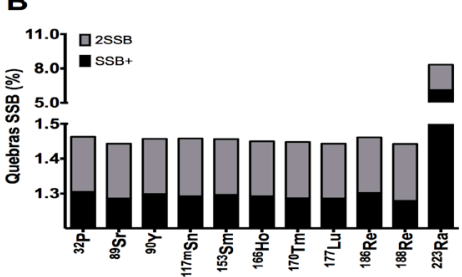

E

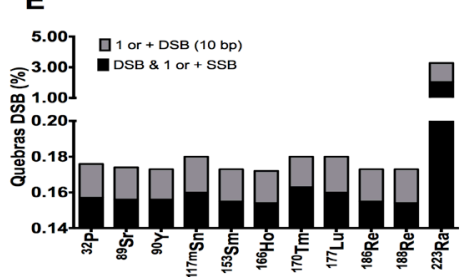

C
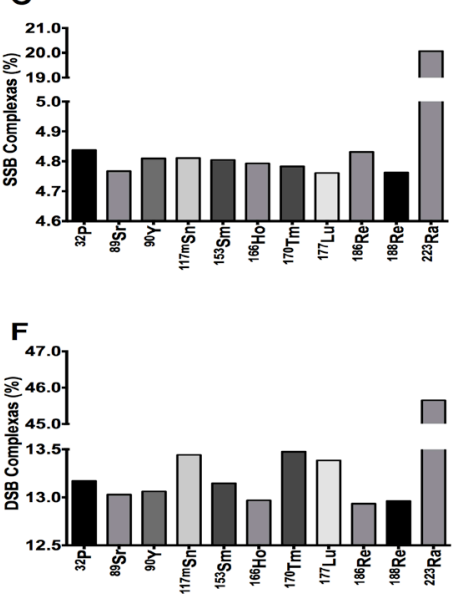

Figure 1. DNA damage results obtained using the MCDS simulator. (A) Percentage of total DNA radioinduced SSB after irradiation; (B) Percentage of two different types of complex SSB; (C) Fraction of complex SSB DNA damages after irradiation; (D) Percentage of total DNA radioinduced DSB after irradiation; (E) Percentage of two different types of complex DSB, (F) Fraction of complex DSB DNA damages after irradiation. 


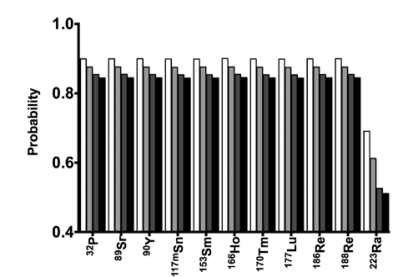

C

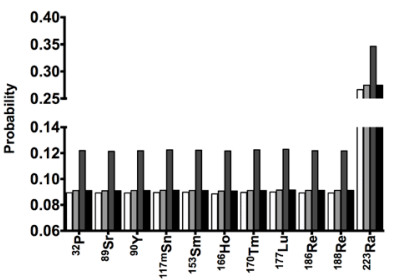

B
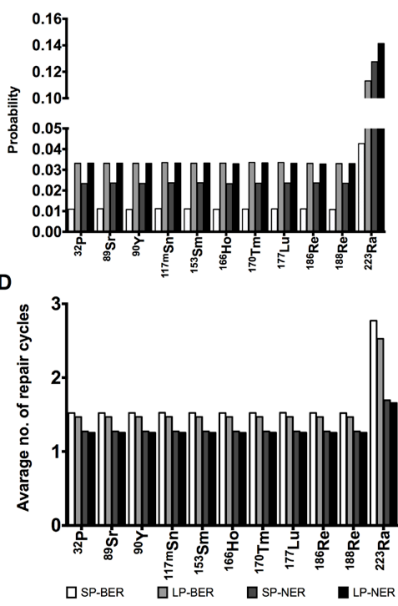

Figure 2. DNA damage repair results obtained using the MCER simulator. Probability (A) of correct repair ( $p$ COR), (B) repair with mutation (p MUT), (C) conversion to DSB (p DSB) of DNA SSB and (D) average number of repair cycles.

\section{DISCUSSION AND CONCLUSION}

Here, different computational methods were used to evaluate multiple radioisotopes for the palliative treatment of bone metastases, specifically in the context of metastatic prostate tumors. In this analysis, radioisotopes currently used in the clinical practice or under research as potentially useful agents for treatment of palliative bone metastases were included. The biological effects resulting from irradiation with these radioisotopes, both at the DNA level and at the cellular level, were evaluated and compared. Our findings indicated that the most promising agents for palliative treatment of bone metastases from prostate cancer are the ${ }^{223} \mathrm{Ra} \alpha$ particles, the ${ }^{177} \mathrm{Lu} \beta^{-}$particles and the ${ }^{170} \mathrm{Tm} \beta^{-}$particles. Other commonly used radioisotopes for palliative treatment of bone metastases, such as ${ }^{89} \mathrm{Sr}$, were found to be inferior in inducing lethal damages than ${ }^{177} \mathrm{Lu},{ }^{170} \mathrm{Tm}$ or ${ }^{223} \mathrm{Ra}$, with radiobiological properties similar to ${ }^{32} \mathrm{P}$. Overall data also suggested that ${ }^{153} \mathrm{Sm} \beta^{-}$particles were superior at inducing cell death compared with ${ }^{89} \mathrm{Sr} \beta^{-}$particles.

The MCDS results showed that the percentage of SSB and DSB after irradiation were highest for ${ }^{223} \mathrm{Ra} \alpha$ particles, followed by ${ }^{177} \mathrm{Lu} \beta^{-}$particles. The same trend was observed for a percentage of complex SSB and DSB. Conversely, the probability of correct repair was low for these irradiating agents and the number of repair cycles was high for all the repair processes studied, regardless of the repair route (MCER results). A higher number of repair cell cycles has been correlated with prolonged repair times [22], [25], which correlates with the increased percentage of complex damages. Previous studies have demonstrated that the complex damage repair by excision can lead to an increased number of DSBs [22], [26]. It is well known that DNA DSBs are frequently associated with apoptosis induction [18]. The damage induced by ${ }^{223} \mathrm{Ra} \alpha$ particles and ${ }^{177} \mathrm{Lu} \beta^{-}$particles seem to have hampered the successful repair of the DNA by BER or NER, presenting a high probability for DSB formation and low probability for correct repair. Furthermore, the high number of DNA DSBs

induced by the ${ }^{223} \mathrm{Ra} \alpha$ particles and ${ }^{177} \mathrm{Lu} \beta^{-}$particles together with their high DNA SSB conversion to DSB may translate an increased probability of apoptosis induction for these three radioisotopes compared with the other investigated agents. In fact, data from the VC simulator estimated a low survival fraction for ${ }^{223} \mathrm{Ra} \alpha$ particles and ${ }^{177} \mathrm{Lu} \beta^{-}$particles, suggesting these irradiating agents would be more promising in the killing of metastatic cells than the other investigated irradiating agents. Furthermore, the VC simulator estimated a low survival fraction for cells irradiated with ${ }^{170} \mathrm{Tm} \beta^{-}$particles. Interestingly, the total number of DSB induced by DNA irradiation with ${ }^{170} \mathrm{Tm} \beta^{-}$ particles was in the low range, but the percentage of complex DSB was ranked together with ${ }^{177} \mathrm{Lu} \beta^{-}$particles, which supports for the importance of modeling the fraction of complex DSB when determining the radiation induced cell damages using radiobiological models.

Detailed analysis of the cellular kinetics post-irradiation using ${ }^{177} \mathrm{Lu} \beta^{-},{ }^{170} \mathrm{Tm} \beta^{-}$and ${ }^{223} \mathrm{Ra} \alpha$ particles showed that the cell response was dose- and radionuclide-dependent. For low dose TCUTs, virtually no differences were found between ${ }^{170} \mathrm{Tm} \beta^{-}$and ${ }^{177} \mathrm{Lu} \beta^{-}$particles. Conversely, for high TCUT values, irradiation with ${ }^{170} \mathrm{Tm} \beta^{-}$particles resulted in a lower number of surviving cells than irradiation with ${ }^{177} \mathrm{Lu} \beta^{-}$particles. This may be a result of differences in the physical half-life of ${ }^{177} \mathrm{Lu}$ and ${ }^{170} \mathrm{Tm}(6.2$ and 127.8 days, respectively) that will contribute differently for dissimilar radiation fractioning.

The findings also showed that a larger number of metastatic cells survived irradiation when compared with normal prostatic cells, in particular, when all cells were actively dividing. This observation highlights the influence of cell proliferation rate on cell response to irradiation, where a faster cell cycle time means the cells were less radiosensitive. Due to its shorter doubling time (54 days versus 500 days for metastatic and normal cells, respectively), metastatic prostate cells were able to compensate radiation-induced damage by rapid cell duplication.

${ }^{223} \mathrm{Ra} \alpha$ particle emission is an appealing strategy for the treatment of bone micrometastases, owing to the short tissue range penetration associated with these radioactive

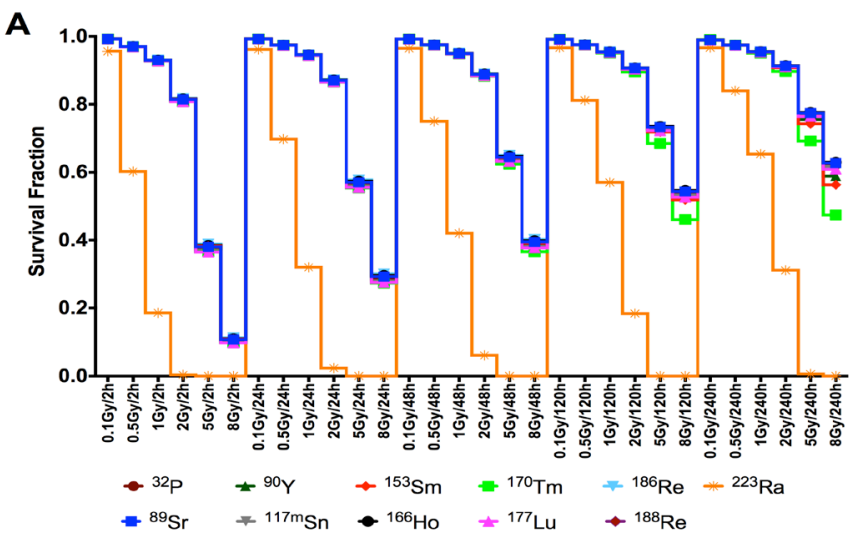

Figure 3. Cell survival fraction estimated for all isotopes in different exposure scenarios. 


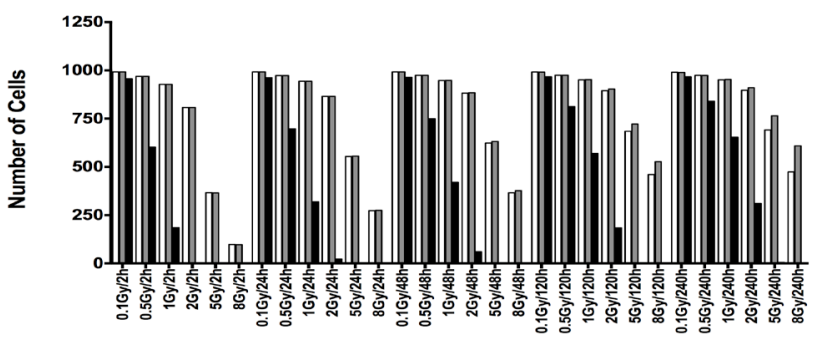

B

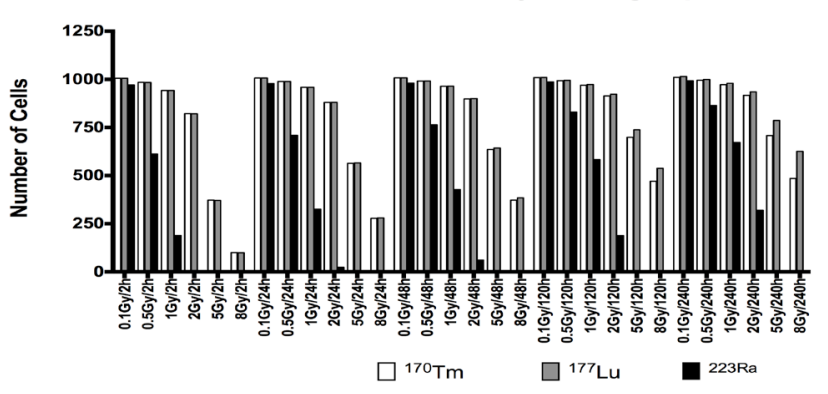

Figure 4. Number of normal prostate cells that survive irradiation with different irradiating agents in a quiescent cell population (A), and in cells actively dividing (B). Simulated doses of $0.1,0.5,1,2,5$ and 8 Gy and irradiation periods of $2,24,48,120$ and $240 \mathrm{~h}$.

particles that can allow for a more circumscribed irradiation surface. Furthermore, typically $\alpha$ emitters induce less hematologic toxicity for a given bone surface dose than $\beta^{-}$emitters. The high linear energy transfer of $\alpha$ particles has been associated with greater biological effectiveness than $\beta^{-}$particles [6], [14], [27]. Our findings indicated that ${ }^{223} \mathrm{Ra} \alpha$ particles induced significantly more DSBs and were more effective in inducing cell death than all the other particles.

Within the $\beta^{-}$particle emitters investigated here, ${ }^{177} \mathrm{Lu}$ has the lowest tissue penetration range $(1.8 \mathrm{~mm})$. A common concern associated with the use of $\beta^{-}$particle emitters is related to its large tissue penetration range of several millimeters, which can result in energy deposition in neighboring, nontargeted cells, a phenomenon known as "crossfire" that may lead to bystander effects. With $\alpha$ particle emitters, due to their short tissue penetration range, the "crossfire" effect is not such a concern, but may require targeting virtually every cell within large size tumors and does not take advantage of the positive bystander effects [28]. The short tissue penetration range of ${ }^{177} \mathrm{Lu} \beta^{-}$particles is placed in the lowest end of the $\beta^{-}$particle emitter's tissue penetration range, minimizing the negative "crossfire" effects often associated with $\beta^{-}$particle emitters; and it is higher than typical $\alpha$ particles allowing us to take advantage of the positive bystander effects that are not attainable with conventional $\alpha$ particles emitters. In addition, ${ }^{177} \mathrm{Lu}$ low $\beta^{-}$particle energy and intermediate physical half-life of 6.73 days allow for a constant dose rate in the target lesion sites [29]. Here, we found that ${ }^{177} \mathrm{Lu} \beta^{-}$particles induced the maximum number of DSB among all $\beta^{-}$emitters studied radionuclides and was part of the top three most effective radionuclides to induce cell death. Data presented here together with the physical characteristics of ${ }^{177} \mathrm{Lu}$ previously described, suggest that replacing the radionuclides ${ }^{153} \mathrm{Sm}$ and ${ }^{89} \mathrm{Sr}$ with
A

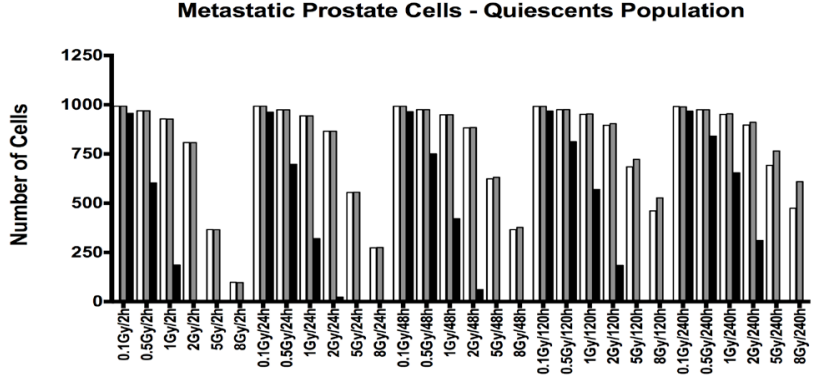

B

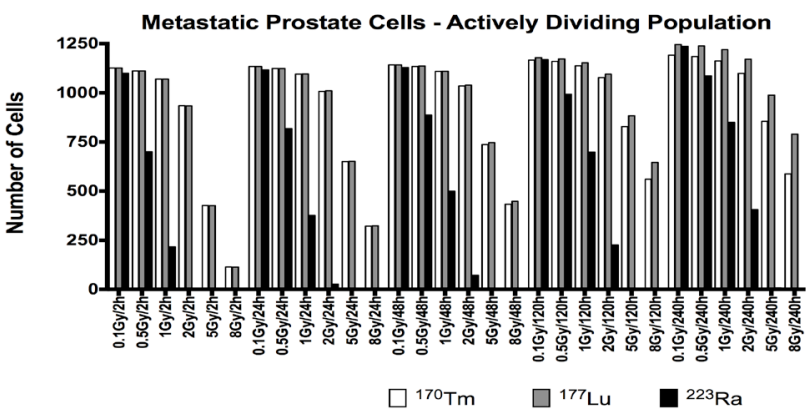

Figure 5. Number of metastatic prostate cells that survive irradiation with different irradiating agents in a quiescent cell population (A), and in cells actively dividing (B). Simulated doses of $0.1,0.5,1,2,5$ and 8 Gy and irradiation periods of $2,24,48,120$ and $240 \mathrm{~h}$

${ }^{177} \mathrm{Lu}$ in the EDTMP complex would improve the potential for cell death in target tissue, while reducing the bone marrow toxicity due to the shorter tissue penetration range of ${ }^{177} \mathrm{Lu} \beta^{-}$ particles in comparison with ${ }^{153} \mathrm{Sm}$ and ${ }^{89} \mathrm{Sr} \beta^{-}$particles.

The long physical half-life of ${ }^{170} \mathrm{Tm}$ (128.6 days) can be an advantage for this radiotracer distribution and storage compared with either ${ }^{223} \mathrm{Ra}$ (11.4 days) or ${ }^{177} \mathrm{Lu}$ (6.2 days) [12]. However, the physical half-life of a radioisotope and the dosing regimen adopted can have a considerable impact on cellular response to irradiation. For example, delivery of $90 \%$ of the total dose of radiation requires approximately 3.5 half-lives of decay, which will correspond to a time interval of approximately 64 weeks for ${ }^{170} \mathrm{Tm}, 6$ weeks for ${ }^{223} \mathrm{Ra}$ and 3 weeks for ${ }^{177} \mathrm{Lu}$ [12]. This may explain the VC results presented here, where the number of cells surviving irradiation with ${ }^{170} \mathrm{Tm} \beta^{-}$particles was lowest when the TCUT was highest.

Computational methods allow for rapid and easy data collection. Nonetheless, some limitations have been pointed out, including modeling and evaluation based on current knowledge, which works as a mechanistic process. Furthermore, the computational algorithms used in this study only model simple repair processes and exclude DSBs repair processes, such as homologous repair and nonhomologous end joining (NHEJ) repair processes. Moreover, the used algorithms assume an optimal scenario of ionizing energy deposition directly into the DNA, scenario that often is challenging to achieve in vitro and in vivo due to inefficient radiotracer biodistribution processes. Therefore, these methods can be useful as a first-line approach to large data sets to help planning subsequent in vitro and in vivo studies, the data presented here requires further validation by means of in vitro and in vivo studies to fully establish the 
therapeutic potential of the investigated radioisotopes in the context of palliative treatment of bone metastases.

In conclusion, this study compared different irradiating agents using the same exposure conditions and controllable cell populations to clarify the quality of these radioisotopes for palliative treatment of bone metastases, specifically in the context of prostate cancer. Collected data allowed the ranking of eleven different radioisotopes in terms of ability to induce DNA damage and probability of damage repair. The top three agents able to induce the highest cellular damage and cell death were found to be the ${ }^{177} \mathrm{Lu} \beta^{-},{ }^{170} \mathrm{Tm} \beta^{-}$and ${ }^{223} \mathrm{Ra} \alpha$ particles. ${ }^{223} \mathrm{Ra}$-dichloride is a particularly appealing approach for the treatment of bone metastases based on the data reported here, this radionuclide physical properties, suitable production methods and feasible logistics of distribution. Within the $\beta^{-}$particle emitters investigated, ${ }^{177} \mathrm{Lu}$ was the preferred one due to its estimated low survival fraction, together with its adequate half-life and physical properties for image acquisition. Data presented here indicated that ${ }^{89} \mathrm{Sr}$ and ${ }^{153} \mathrm{Sm}$ had modest effect on the cell survival fraction. Although in vitro and in vivo data are necessary to support our findings, the observations reported here may support for a careful rethinking of the widespread clinical use of ${ }^{89} \mathrm{Sr}$ and ${ }^{153} \mathrm{Sm}$ and, perhaps, strengthen the use of ${ }^{223} \mathrm{Ra}$ and ${ }^{177} \mathrm{Lu}$ in the palliative treatment of bone metastases.

\section{REFERENCES}

[1] B. W. Stewart and C. P. Wild, World Cancer Report 2014. IARC Nonserial Publication, 2014, p. 630.

[2] S. Chakraborty, T. Das, H. D. Sarma, M. Venkatesh, and S. Banerjee, "Comparative studies of 177Lu-EDTMP and $177 \mathrm{Lu}-$ DOTMP as potential agents for palliative radiotherapy of bone metastasis.," Appl. Radiat. Isot., vol. 66, no. 9, pp. 1196-205, Sep. 2008.

[3] C. L. Maini, R. Sciuto, L. Romano, and S. Bergomi, "Radionuclide therapy with bone seeking radionuclides in palliation of painful bone metastases.," J. Exp. Clin. Cancer Res., vol. 22, no. 4 Suppl, pp. 71-4, Dec. 2003.

[4] M. Bedi, D. M. King, and S. Tutton, "Minimally Invasive Local Treatments for Bone and Pulmonary Metastases," Minim. Invasive Surg., pp. 1-9, 2014.

[5] V. J. Lewington, "Bone-seeking radionuclides for therapy.," J. Nucl. Med., vol. 46 Suppl 1, no. 1, p. 38S-47S, Jan. 2005.

[6] N. Pandit-Taskar, S. M. Larson, and J. a Carrasquillo, "BoneSeeking Radiopharmaceuticals for Treatment of Osseous Metastases, Part 1: $\alpha$ Therapy with 223Ra-Dichloride.," J. Nucl. Med., vol. 55, no. 2, pp. 268-74, Feb. 2014.

[7] E. B. Silberstein and S. M. Drive, "Dosage and Response in Radiopharmaceutical Therapy of Painful Osseous Metastases Dosage and Response in Radiopharmaceutical Therapy of," J. Nucl. Med., vol. 37, pp. 249-252, 1996.

[8] M. R. a. Pillai, S. Chakraborty, T. Das, M. Venkatesh, and N. Ramamoorthy, "Production logistics of $177 \mathrm{Lu}$ for radionuclide therapy," Appl. Radiat. Isot., vol. 59, no. 2-3, pp. 109-118, Aug. 2003.

[9] F. J. Daha, el al., "Production of $177 \mathrm{Lu}$ and formulation of Ethylene diamine tetramethylene phosphonate (EDTMP) kits as a bone-seeking radiopharmaceutical," Iran. J. Radiat. Res., vol. 7, no. 4, pp. 229-234, 2010.

[10] C. Wang, J. Wang, H. Jiang, M. Zhu, B. Chen, and W. Bao, "In vitro study on apoptosis induced by strontium-89 in human breast carcinoma cell line.," J. Biomed. Biotechnol., vol. 2011, p. 541487, Jan. 2011.
[11] L. G. Bouchet, W. E. Bolch, S. M. Goddu, R. W. Howell, and D. V Rao, "Considerations in the selection of radiopharmaceuticals for palliation of bone pain from metastatic osseous lesions.," $\mathrm{J}$. Nucl. Med., vol. 41, no. 4, pp. 682-7, Apr. 2000.

[12] O. Sartor, "Overview of samarium sm153 lexidronam in the treatment of painful metastatic bone disease.," Rev. Urol., vol. 6 Suppl 10, Jan. 2004.

[13] T. Das, S. Chakraborty, H. D. Sarma, P. Tandon, S. Banerjee, M. Venkatesh, and M. R. a Pillai, "(170)Tm-EDTMP: a potential costeffective alternative to (89) $\mathrm{SrCl}(2)$ for bone pain palliation.," $\mathrm{Nucl}$. Med. Biol., vol. 36, no. 5, pp. 561-8, Jul. 2009.

[14] M. R. Harrison, T. Z. Wong, A. J. Armstrong, and D. J. George, "Radium-223 chloride: a potential new treatment for castrationresistant prostate cancer patients with metastatic bone disease.," Cancer Manag. Res., vol. 5, pp. 1-14, Jan. 2013.

[15] J. Goyal and E. Antonarakis, "Bone-targeting radiopharmaceuticals for the treatment of prostate cancer with bone metastases," Cancer Lett., vol. 323, no. 2, pp. 135-146, 2012.

[16] F. D. C. Guerra Liberal, A. A. S. Tavares, and J. M. R. S. Tavares, "Comparative analysis of 11 different radioisotopes for palliative treatment of bone metastases by computational methods," Med. Phys., vol. 41, no. 114101, 2014.

[17] V. a Semenenko and R. D. Stewart, "A fast Monte Carlo algorithm to simulate the spectrum of DNA damages formed by ionizing radiation.," Radiat. Res., vol. 161, no. 4, pp. 451-7, Apr. 2004.

[18] D. J. Carlson, R. D. Stewart, V. a Semenenko, and G. a Sandison, "Combined use of Monte Carlo DNA damage simulations and deterministic repair models to examine putative mechanisms of cell killing.," Radiat. Res., vol. 169, no. 4, pp. 447-59, Apr. 2008.

[19] H. Nikjoo, S. Uehara, W. E. Wilson, M. Hoshi, and D. T. Goodhead, "Track structure in radiation biology: theory and applications.," Int. J. Radiat. Biol., vol. 73, no. 4, pp. 355-64, Apr. 1998.

[20] H. Nikjoo, "Radiation track and DNA damage," Iran. J. Radiat. Res., vol. 1, no. 1, pp. 3-16, 2003.

[21] V. a Semenenko and R. D. Stewart, "Fast Monte Carlo simulation of DNA damage formed by electrons and light ions.," Phys. Med. Biol., vol. 51, no. 7, pp. 1693-706, Apr. 2006.

[22] V. A. Semenenko and R. D. Stewart, "Monte Carlo Simulation of base and nucleotide excision repair of clustered DNA damage sites. II. Comparisons of model predictions to measured data.," Radiat. Res., vol. 164, no. 2, pp. 194-201, 2005.

[23] R. Sach, P. Feld, and D. J. B. Er, "Review The link between low LET dose-response relations and the underlying kinetics of dam age production / repair / m isrepair," Int. J. Radiat. Biol., vol. 72, no. 4, pp. 351-374, 1997.

[24] R. R. Berges, J. et al., "Implication of cell kinetic changes during the progression of human prostatic cancer.," Clin. Cancer Res., vol. 1, no. 5, pp. 473-80, May 1995.

[25] A. A. S. Tavares and J. M. R. S. Tavares, "Evaluating 99mTc Auger electrons for targeted tumor radiotherapy by computational methods," Med. Phys., vol. 37, no. 7, p. 3551, 2010.

[26] V. A. Semenenko, R. D. Stewart, and A. E. J, "Monte Carlo simulation of base and nucleotide excision repair of clustered DNA damage sites. I. Model properties and predicted trends.," Radiat. Res., vol. 164, no. 2, pp. 180-193, 2005.

[27] S. Nilsson, et al., "Two-year survival follow-up of the randomized, double-blind, placebo-controlled phase II study of radium-223 chloride in patients with castration-resistant prostate cancer and bone metastases.," Clin. Genitourin. Cancer, vol. 11, no. 1, pp. 20 6, Mar. 2013.

[28] M. R. Jackson, N. Falzone, and K. a Vallis, “Advances in anticancer radiopharmaceuticals.," Clin. Oncol. (R. Coll. Radiol)., vol. 25, no. 10, pp. 604-9, Oct. 2013.

[29] D. Máthé, et al, "Multispecies animal investigation on biodistribution, pharmacokinetics and toxicity of 177Lu-EDTMP, a potential bone pain palliation agent.," Nucl. Med. Biol., vol. 37, no. 2, pp. 215-26, Feb. 2010. 